\title{
Dinámica de la comunidad de macroinvertebrados en la quebrada Potrerillos (Colombia): Respuesta a los cambios estacionales de caudal
}

\author{
Magnolia Longo S. ${ }^{1,3, *}$, Hilldier Zamora G. ${ }^{1}$, Cástor Guisande G. ${ }^{2}$ y John Jairo Ramírez R. ${ }^{3}$ \\ ${ }^{1}$ Facultad de Ciencias Naturales, Exactas y de la Educación, Universidad del Cauca. Popayán, Colombia. \\ ${ }^{2}$ Facultad de Ciencias, Universidad de Vigo, Lagoas-Marcosende. 36200-Vigo, España. \\ ${ }^{3}$ Facultad de Ciencias Exactas y Naturales, Universidad de Antioquia. A.A. 9634. Medellín, Colombia. \\ * Autor responsable de la correspondencia: mc_longo@ hotmail.com
}

Recibido: 14/7/09

Aceptado: 24/2/10

\begin{abstract}
Macroinvertebrate community dynamics in the Potrerillos stream (Colombia): Response to seasonal flow changes

Between January and December 2006 monthly samplings of benthic macroinvertebrates in two sampling stations were conducted in the Potrerillos stream. This article presents the analysis of the spatial and temporal changes in the composition and structure of this community as a function of the seasonality of rains in a drywood tropical region. It was determined that the periods of scant and moderate rains were beneficial to processes of colonization and development of different taxa, as these periods presented the highest values of abundance, richness and diversity $\left(H^{\prime}\right)$. This last concept was related to a continuous flow of low variability, which favored the heterogeneity of habitats, favorable levels of physical and chemical variables, and the availability of higher food supply. During the dry season, the flow ceased and several isolated ponds were formed, habitats and food were lost, physical and chemical conditions were altered, many taxa disappeared and the taxa best adapted to lenitic zones and moist substrates were dominant. The abundant rains brought new hydrologic dynamics, which, though they generated unstable habitats and microhabitats, allowed for the colonization of organisms, mainly those with winged instars, which increased richness and diversity. Despite that disturbances caused by drought and abundant rains caused fluctuations in the habitats' characteristics and in the composition and structure of the community, the ecosystem was stable and resilient with reference to these community attributes.
\end{abstract}

Key words: Tropical seasonal stream, stability, drought, aquatic macroinvertebrates, diversity.

\section{RESUMEN}

Dinámica de la comunidad de macroinvertebrados en la quebrada Potrerillos (Colombia): Respuesta a los cambios estacionales de caudal

Entre enero y diciembre de 2006 se llevaron a cabo muestreos mensuales de macroinvertebrados bentónicos en dos estaciones de muestreo en la quebrada Potrerillos. Este artículo presenta el análisis de los cambios espaciales y temporales en la composición y estructura de esta comunidad en función de la estacionalidad de las lluvias en una región de bosque seco tropical. Se determinó que los períodos de lluvia escasa y moderada beneficiaron procesos de colonización y desarrollo de diversos taxa, presentando estas épocas los valores más altos de abundancia, riqueza y diversidad $\left(\mathrm{H}^{\prime}\right)$. Lo anterior se relacionó con un caudal continuo y poco variable, que favoreció la heterogeneidad de hábitats, niveles propicios de variables físicas y químicas y la disponibilidad de mayor oferta alimenticia. En la época de sequía cesó el caudal y se formaron pozos aislados, hubo pérdida de hábitats y de alimento, se alteraron las condiciones fisicoquímicas, desaparecieron muchos taxa y dominaron los mejor adaptados a zonas leníticas y sustratos húmedos. Las lluvias abundantes trajeron una nueva dinámica hídrica, que aunque generaron hábitats y microhábitats inestables, permitió la colonización de organismos, principalmente de aquellos con estadios alados, lo que incrementó la riqueza y diversidad. A pesar que los disturbios por la sequía y las lluvias abundantes ocasionaron fluctuaciones en las características de los hábitats y en la composición y estructura de la comunidad, el ecosistema fue estable y resiliente en lo referente a estos atributos comunitarios.

Palabras clave: Quebrada estacional tropical, estabilidad, sequía, macroinvertebrados acuáticos, diversidad. 


\section{INTRODUCCIÓN}

Un caso particular en la ecología de los sistemas lóticos es el de las quebradas y arroyos intermitentes. Estos ecosistemas, sometidos a la estacionalidad de las descargas hídricas, presentan una fauna de macroinvertebrados y peces excepcional en composición y estructura. Los organismos que allí habitan responden para sobrevivir a una serie de disturbios ocasionados por las sequias y las inundaciones. La variabilidad en el régimen de caudales influye, como lo han planteado Junk \& Wantzen (2004), en la estabilidad, diversidad y disponibilidad de hábitats, en la morfometría fluvial y en la conectividad lateral de los flujos de agua con las zonas inundables, alterándose además la conexión longitudinal del canal -"río discontinuo" (Ward \& Stanford, 1983)- y la conectividad vertical con las zonas hiporréicas (Boulton, 2003). Para Poole (2002), estas características pueden ser más influyentes en el normal desarrollo y estabilidad de una especie y su comunidad que las presentadas en los ríos continuos.

En este sentido, no solo los ecosistemas acuáticos de las regiones templadas están sujetos a variaciones en los regímenes hidrológicos, sino también los ecosistemas de climas cálidos, principalmente los de zonas con altas temperaturas y bajas precipitaciones, en donde también ocurren sequías con diversos grados de intensidad y duración y, por tanto, con diversas implicaciones ecológicas para los organismos (Covich et al., 2003; Ramírez et al., 1998).

La sequía -más allá de la connotación hidráulica y cultural como "un período de flujo bajo imprevisible", excepcional en duración, extensión, severidad o intensidad (Humphries \& Baldwin, 2003)- desde el punto de vista ecológico y de sus repercusiones en los ecosistemas acuáticos, se cataloga como: 1) sequía estacional, que es predecible, típica de climas Mediterráneos y Húmedos-Secos Tropicales; y 2) sequía supraestacional, imprevisible, con períodos de sequía más prolongados, y que probablemente se ajusta más a las definiciones tradicionales de sequía (Lake, 2003). De acuerdo con Boulton (2003), esta distinción ha sido esencial ya que la sequía estacional es propia del régimen hidrológico en ciertas zonas y en ciertos períodos del año, razón por la cual, muy probablemente gran parte de la biota no sufre mayores alteraciones en sus ciclos de vida.

En ríos y quebradas del Mediterráneo se ha encontrado que la fauna de macroinvertebrados presenta ensambles estacionales sincronizados con los cambios hidrológicos y correlacionados con variables microclimáticas, geológicas o del sustrato [e.g. regiones Mediterráneas en general (Gasith \& Resh, 1999) y Norte de África (Arab et al., 2004)] y con las actividades antrópicas [Bonada (2003) en el Mediterráneo; Arab et al. (2004)]. Además, tanto para ecosistemas templados como mediterráneos, se informa que los ríos intermitentes en comparación con los perennes, presentan menor abundancia y riqueza de macroinvertebrados debido a los disturbios ocasionados por la sequía y las lluvias abundantes (Halwas et al., 2005). Entre dichos disturbios se encuentran las alteraciones del hábitat físico (tales como ancho activo, profundidad, velocidad y sedimentación), de las condiciones químicas (Wiggins, 1973; Tramer, 1977; Mol et al., 2000) y de los factores biológicos (como competencia y predación) (Williams, 1996; Statzner \& Higler, 1986; Boulton, 2003).

En algunos ecosistemas Neotropicales ocurren fenómenos similares a los anteriores: baja diversidad de especies con alto número de especies raras y poca abundancia [e.g. Brasil: Ribeiro \& Uieda (2005)]; predominio de Chironomidae (Diptera) en las épocas de alto y bajo flujo [e.g. Ribeiro \& Uieda (2005); Venezuela: Rincón \& Cressa (2000)], preponderancia de Philopotamidae (Trichoptera) durante la sequía y de Leptophlebiidae y Baetidae (Ephemeroptera) durante las épocas de mayor lluvia (Rincón \& Cressa, 2000). En Puerto Rico, con base en un estudio de seis años (1992-1998) sobre poblaciones de Atya lanipes (Holthuis 1963) y Xiphocaris elongata (Guérin-Méneville 1955) (Decapoda), se encontró que sequias prolongadas alteraron significativamente las comunidades acuáticas, debido a la contracción de los hábitats y a la disminución prolongada del rendimiento reproductor (Covich et al., 2003).

Aunque se reconoce ampliamente que la duración de las épocas secas es un factor determinante 


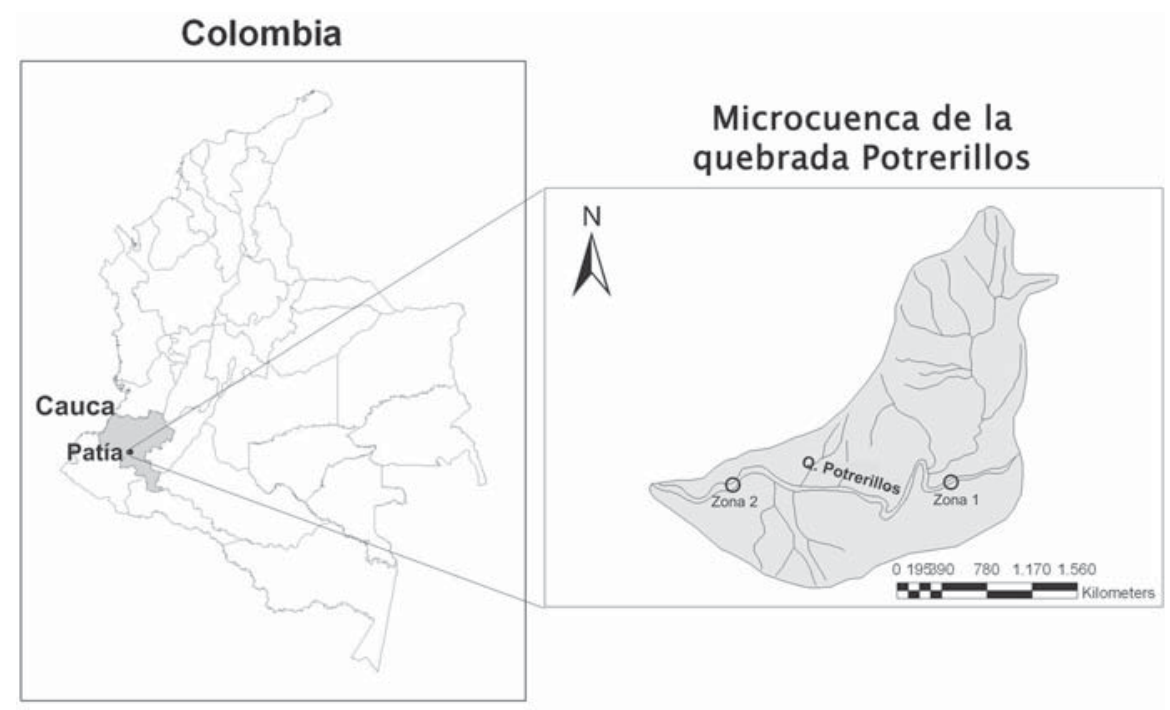

Figura 1. Localización de las zonas de muestreo en la quebrada Potrerillos, Valle geográfico del río Patía (Cauca-Colombia). Location of the sampling zones in the Potrerillos stream, geographic valley of the Patía river (Cauca-Colombia).

en la biodiversidad, los estudios en el Neotrópico son escasos y, como se ha comprobado en otros casos, los procesos y patrones que ocurren en estas zonas no necesariamente se ajustan a las teorías propuestas sobre ecología de ecosistemas lóticos en zonas templadas [e.g. Concepto del río Continuo de Vannote et al. (1980) y grupos tróficos funcionales de macroinvertebrados de Merrit \& Cummins (1996) y Tomanova et al. (2006)].

Fundamentados en lo anterior, nos preguntamos cómo varían temporal y espacialmente la composición y estructura de la comunidad de macroinvertebrados acuáticos en función del estrés representado por la variación estacional en el nivel del agua en la quebrada Potrerillos. Se predice que durante la época seca disminuye la diversidad de taxa y aumenta la densidad de organismos con estrategias para resistir la desecación (moluscos y dípteros), y a su vez disminuyen los Ephemeroptera, Trichoptera y ciertos Coleoptera. Por otra parte, si entre zonas hay diferencias en las actividades antrópicas y la cobertura de la vegetación ribereña, entonces habrá diferencias de diversidad y composición entre ellas. Con el fin de corroborar estas hipótesis se evalúan los cambios en la composición y estructura de la comunidad tanto espacial como temporalmente y, se establece su relación con los cambios hidrológicos en la quebrada.
Se escogió la quebrada Potrerillos porque se encuentra incluida en el $0.7 \%$ del territorio nacional que presenta proceso de desertificación acelerado, por lo que es importante conocer la dinámica de los componentes bióticos y abióticos de dicho ecosistema, que además es influenciado por el ciclo El Niño-La Niña-Oscilación del Sur (4-7 años), por la orografía del territorio y por actividades antrópicas con un grado de intervención medio (Ministerio de Ambiente, Vivienda y Desarrollo Territorial, 2004).

\section{MATERIAL Y MÉTODOS}

\section{Área de muestreo}

La quebrada Potrerillos se ubica en la región norte del valle geográfico del río Patía, al sur del departamento del Cauca, en el municipio El BordoPatía (Fig. 1). El valle, con altitudes entre 600 y $700 \mathrm{msnm}$, está conformado por terrazas aluviales onduladas, enmarcadas por las cordilleras Central y Occidental (Lemus, 1990). Es una zona de vida transicional entre bosque húmedoPremontano (bh-PM) y bosque seco-Basal Tropical (bs-BT) según Holdridge (1978). Presenta un clima cálido entre subhúmedo y seco, temperatu- 
ra media anual de $28.1^{\circ} \mathrm{C}$, con máximas absolutas de $38.5^{\circ}$ (de julio a septiembre). El régimen anual de lluvias es bimodal, comprendido entre los meses de octubre a diciembre y de marzo a mayo, siendo noviembre el mes más lluvioso con un promedio de $290 \mathrm{~mm}$. El periodo seco se presenta entre junio y septiembre, siendo el mes más seco julio con 43 mm (IDEAM, 1999).

Potrerillos es una corriente de segundo orden, de aguas claras, con un área de captación de $41.71 \mathrm{~km}^{2}$ y una longitud promedio de $8.0 \mathrm{~km}$. El lecho fluvial está constituido por arenas, arcillas, gravas y cantos redondeados, cuyos tamaños alcanzan hasta $30 \mathrm{~cm}$ de diámetro; también se encuentran algunas rocas localizadas (Torres et al., 1992).

Este estudio se efectuó en dos zonas de la quebrada: Zona 1 (Z1), parte alta (700 msnm), ubicada a $2^{\circ} 07^{\prime} 47.7^{\prime \prime} \mathrm{N}$ y $77^{\circ} 00^{\prime} 41.8^{\prime \prime} \mathrm{W}$; y Zona 2 (Z2), parte baja (613 msnm), ubicada a $2^{\circ} 06^{\prime} 17.1^{\prime \prime} \mathrm{N}$ y $77^{\circ} 04^{\prime} 13.6^{\prime \prime} \mathrm{W}$, a $2 \mathrm{~km}$ de la desembocadura al río Patía (Fig. 1). El ancho promedio del cauce durante la época de mayor pluviosidad fue de $13.4 \mathrm{~m}$ y su profundidad máxima de $1 \mathrm{~m}$. LaZ1 presentó mayor cobertura de bosques ribereños (85\%), conformados principalmente por Acacia farnesiana y Achatocarpus nigricans; en la Z2, predominaron matorrales arbustivos de tipo subxerofíticos con baja cobertura $(30 \%)$.

\section{Variables físicas y químicas}

Los registros de las variables físicas y químicas y el muestreo de macroinvertebrados se realizaron mensualmente, desde enero hasta diciembre de 2007, exceptuando abril y mayo.

La quebrada presentó drásticas variaciones en el flujo de la corriente en relación con el régimen pluviométrico; consecuentemente, durante la época seca se registró una disminución progresiva del ancho del cauce, lo que ocasionó la formación de algunos pozos aislados que contenían una alta proporción de materiales orgánicos, particularmente hojarasca. Por el contrario, en la época de lluvias abundantes se presentaron crecidas de diversa intensidad que homogenizaron los hábitats, eliminaron los pozos y formaron rápidos.

Para la medición de las variables químicas durante las épocas de lluvia se colectó in situ en cada zona 1 litro de agua, compuesto por submuestras provenientes de tres puntos tomados al azar del área transversal del lecho. Durante la sequía se recogió el agua de uno o dos pozos.

Para la estimación en el laboratorio de algunas variables químicas se tomó 1 litro de agua en envases plásticos etiquetados, almacenados y refrigerados $\left(4-5^{\circ} \mathrm{C}\right)$ durante 24 horas. Las variables

Tabla 1. Variables físicas y químicas registradas en la quebrada Potrerillos (Cauca-Colombia). Physical and chemical variables registered in the Potrerillos stream (Cauca-Colombia).

\begin{tabular}{lcc}
\hline \multicolumn{1}{c}{ Variables } & Unidad & Material y/o Método \\
\hline Pluviosidad & $\mathrm{mm}$ & Pluviómetro (IDEAM) \\
$*$ Caudal & $\mathrm{m}^{3} / \mathrm{s}$ & Molinete (Kempten, serie C2132287) \\
$*$ Temperatura del agua & ${ }^{\circ} \mathrm{C}$ & Termómetro \\
$*$ Conductividad & $\mu \mathrm{mhos} / \mathrm{cm}$ & Potenciométrico (Conductímetro) \\
Turbiedad & $\mathrm{NTU}$ & Espectrofotométrico \\
$*$ Oxígeno disuelto & $\mathrm{mg} / 1$ & Potenciométrico (Oxímetro) \\
$*$ Dióxido de carbono & $\mathrm{mg} / 1$ & NaOH y fenolftaleína (Titulación) \\
$*$ pH & $\mathrm{Unidades}$ & Potenciométrico (pH-metro) \\
$*$ Alcalinidad total & $\mathrm{mg} \mathrm{CaCO} / 1$ & A partir de pH y CO 2 \\
$*$ Durezas total y carbonácea & $\mathrm{mg} \mathrm{CaCO} / 1$ & Kit Merck \\
Amonio & $\mathrm{mg} / 1$ & Espectrofotométrico \\
Nitritos & $\mathrm{mg} / 1$ & Espectrofotométrico \\
Nitratos & $\mathrm{mg} / 1$ & Espectrofotométrico \\
\hline
\end{tabular}

\footnotetext{
* variables medidas in situ
} 
físicas, químicas y los materiales y métodos utilizados para su medición se presentan en la Tabla 1.

\section{Macroinvertebrados bentónicos}

Para garantizar una mayor representatividad de la composición y estructura de la comunidad estudiada, se colectaron en cada zona tres muestras, una por microhábitat (cantos, hojarasca y vegetación ribereña) empleando una red Surber (área $\left.0.16 \mathrm{~m}^{2}, \phi: 0.4 \mathrm{~mm}\right)$. Aunque cada muestra se procesó por separado, para los análisis los registros de abundancia fueron promediados y considerados como un solo dato para cada fecha y zona.

Cuando cesó el flujo hídrico, para buscar individuos en el hiporreo, se extrajo en cada zona una muestra de arena utilizando un tubo $(\phi: 10 \mathrm{~cm})$ con un metro de longitud. La muestra obtenida fue depositada en una bandeja blanca de la cual se retiraron manualmente los individuos. Todas las muestras fueron fijadas, etiquetadas y transportadas en envases con alcohol al $90 \%$.

Los individuos fueron separados, identificados, contados y almacenados en el laboratorio de Recursos Hidrobiológicos Continentales de la Universidad del Cauca. La identificación se realizó hasta la menor jerarquía taxonómica posible (géneros y morfotipos, excepto para Díptera), utilizando las claves taxonómicas de McCafferty \& Provonsha (1978), Pennak (1978), Merrit \& Cummins (1996), Roldán (1988), Rodríguez et al. (1992) y Fernández \& Domínguez (2001).

\section{Análisis de datos}

\section{Condiciones abióticas de la quebrada}

Para establecer la significancia de las diferencias espaciales y temporales entre las zonas de muestreo con respecto a las épocas pluviométricas y las variables físicas y químicas se aplicó un análisis discriminante. Para ello, se definieron previamente ocho grupos, representando cada uno una zona y una época hidrológica diferente (e.g. grupo 1: Z1-lluvia moderada; grupo 2: Z2-lluvia escasa, grupo 5: Z1-sequía, etc.). Las épocas se definieron con base en el régimen hidrológico, y cada una contenía entre 2 y 4 meses. Con este análisis se estableció si los grupos predefinidos persistían o se asociaban en un nuevo grupo, se precisaron las diferencias entre los conglomerados previamente conformados y, se depuró y redujo el número de variables que determinan esas diferencias entre todo el pool de variables.

\section{Diversidad comunitaria}

La eficiencia de los muestreos se determinó a partir de la comparación entre la riqueza observada y la esperada utilizando el programa EstimateS 6.0 PL (Colwell, 1997). La riqueza esperada se calculó con el estimador Chao 1.

Con el fin de establecer los cambios temporales y espaciales de la composición y estructura de la comunidad se elaboró una tabla de abundancia por taxón para cada fecha y punto de muestreo. Con estos valores se estimaron la riqueza específica y la diversidad alfa $(\alpha)$; esta última se calculó con base en la diversidad de Shannon \& Wiener, la dominancia de Simpson y la equidad de Pielou. Estos estimativos se calcularon con el programa BioDiversityPro v.2. Con base en dichos índices se aplicó un test de Kruskal-Wallis para establecer posibles diferencias significativas espaciales y temporales.

La dinámica de la variación temporal de la abundancia de los macroinvertebrados y de las variables físicas y químicas en cada zona fue determinada mediante el índice de Estabilidad o Fluctuación de Dubois. Para tal efecto, se partió del cálculo individual del índice de estabilidad para la abundancia de cada taxón y para cada variable fisicoquímica -ambas estandarizadas a una escala de 0 a 1 aplicando: $V E=(x-\operatorname{Min}) /(\operatorname{Max}-\operatorname{Min})$, donde $V E$ es el valor estandarizado, Max y Min son los valores máximo y mínimo de cada variable, y $x$ es cada uno de los valores de cada variable- en cada fecha de muestreo. El índice final para la abundancia se obtuvo sumando los valores individuales por taxón; igualmente se hizo con el índice para las variables abióticas (Guisande et al., 2006). Los cálculos se realizaron con SPSS v. 13.0.

Para establecer las variables fisicoquímicas de las cuales depende el índice de fluctuación mensual de la abundancia de los macroinvertebrados, 
los datos fueron analizados mediante un modelo de regresión múltiple por pasos sucesivos (stepwise) utilizando el paquete SPSS v. 13.0. Los datos de las variables fisicoquímicas empleadas para ejecutar el modelo fueron los valores individuales de estabilidad de cada una de ellas.

\section{RESULTADOS}

\section{Variables físicas y químicas}

Conforme con los registros históricos (19791990), la precipitación disminuyó ostensiblemente durante el período de este estudio a causa del fenómeno de El Niño (Ideam, 2007) (Fig. 2). Se destaca el período entre septiembre y finales de octubre, puesto que estos meses, considerados históricamente como períodos de lluvias abun-

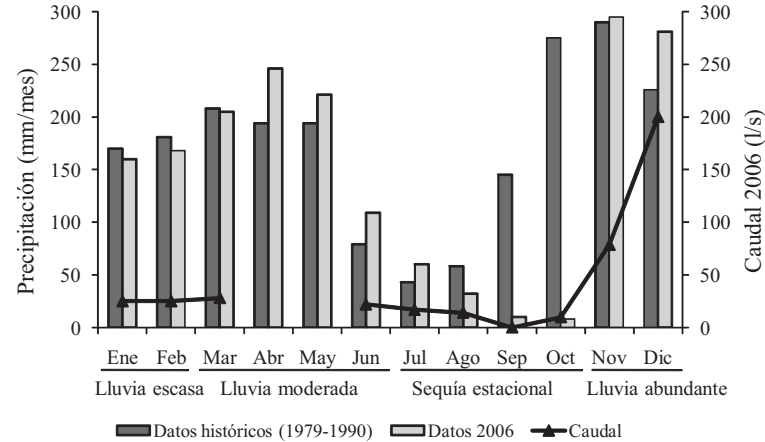

Figura 2. Variación temporal de la precipitación entre 1979 y 1990, durante el año 2006 en el municipio de Patía (CaucaColombia), y variaciones de caudal en la quebrada Potrerillos durante el año 2006 (excepto abril y mayo). La clasificación de las épocas pluviométricas se basó en los registros de 2006. Rainfall temporal variation between 1979 and 1990, during the year 2006 in the Patía municipality (Cauca-Colombia); and flow variations in the Potrerillos stream during the year 2006 (except April and May). The classification of pluviometric periods is based on the 2006 records.
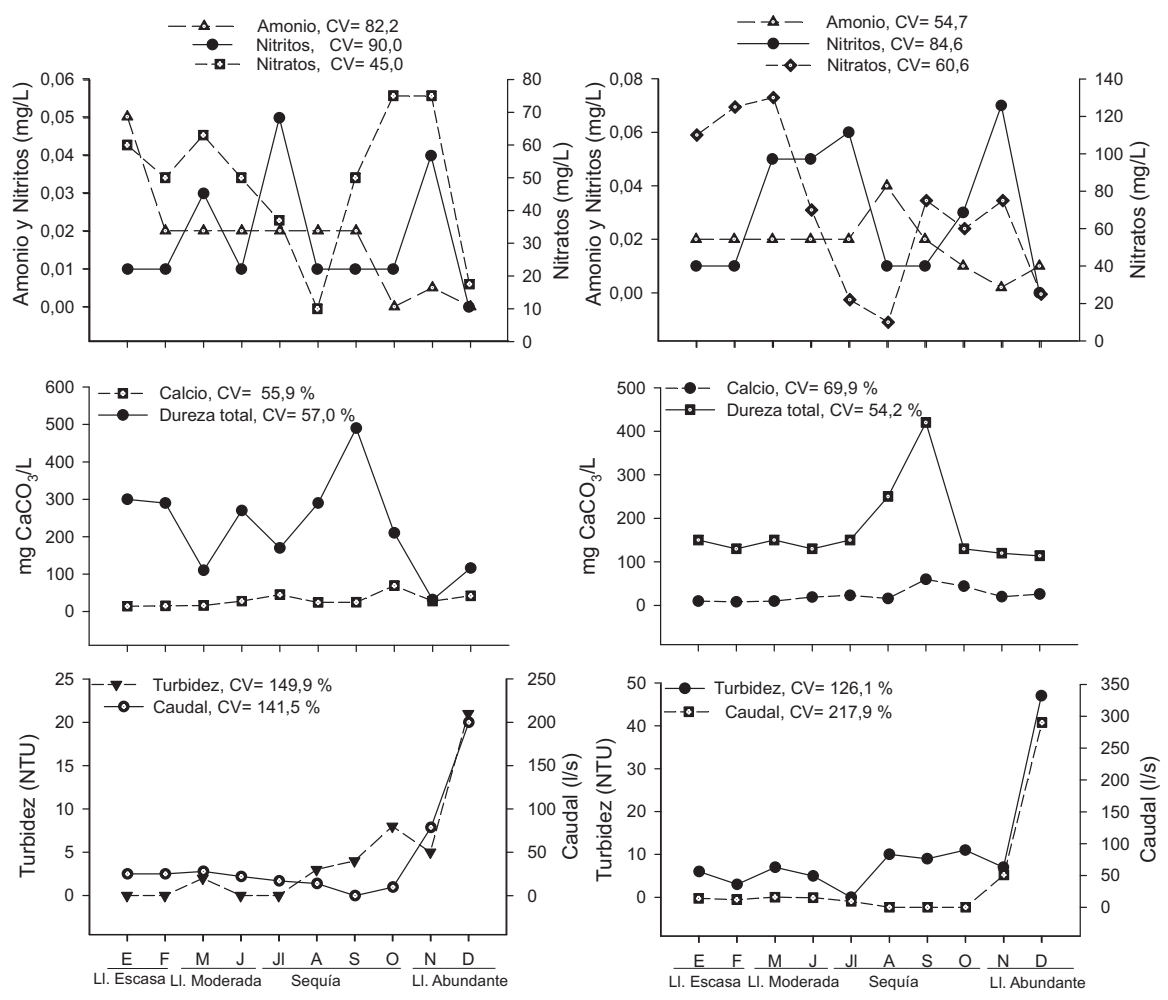

(a)

(b)

Figura 3. Valores mensuales y coeficientes de variación (CV) de amonio, nitritos, nitratos, dureza total, calcio, turbidez y caudal en las zonas 1(a) y 2(b) de la quebrada Potrerillos (Cauca-Colombia). Monthly values and coefficients of variation (CV) for ammonia, nitrites, nitrates, total hardness, calcium, turbidity, and flow in the zones 1(a) and 2(b) of the Potrerillos stream (Cauca-Colombia). 
dantes, en el año 2006 presentaron una sequía estacional prolongada. Durante ésta hubo pérdida significativa y constante del flujo hídrico, con la consecuente formación de algunos pozos aislados, que a su vez perdieron gradualmente profundidad y área de llenado dada la acumulación de sedimentosy materia orgánica particulada. Además, se reportó un continuo aumento del nivel freático.

De acuerdo con la intensidad y frecuencia de los eventos de lluvia se diferenciaron cuatro épocas hidrológicas, correspondientes a: 1) lluvias escasas: enero y febrero, 2) lluvias moderadas: de marzo a junio, 3) sequía estacional: de julio a octubre, y 4) lluvias abundantes: noviembre y diciembre.

En la Z2 se registraron valores más bajos de caudal durante las épocas lluviosas y mayor permanencia de pozos en la sequía (de agosto a octubre) con respecto a la Z1 (septiembre y octubre). Este comportamiento fue consecuencia del incremento en la extracción de agua para actividades agropecuarias, al aumento en el ancho del cauce y a la disminución de la cobertura vegetal, con lo cual se presenta mayor área de exposición para procesos de evaporación.

Las variables físicas y químicas en la quebrada respondieron significativamente a los efectos de la sequía. Las temperaturas ambiental e hídri- ca y el $\mathrm{pH}$ fueron las variables más estables a lo largo del tiempo (CV entre 3.7 y 9.4\%). La saturación de oxígeno disuelto presentó una variación relativamente alta en ambas zonas $\left(\mathrm{CV}_{Z 1}=\right.$ $24.4 \%, \mathrm{CV}_{Z 2}=34.7 \%$ ); en las épocas de lluvia, en los rápidos se registraron porcentajes entre 40 y 100 , y en los pozos se alcanzaron valores de $30 \%$. El $\mathrm{CO}_{2}$ disuelto al igual que los nitritos y nitratos, disminuyó en las épocas de menor flujo, probablemente a causa del incremento en el florecimiento de perifiton (Díaz et al., 2008). Las concentraciones de dureza total y carbonácea, del calcio y de la conductividad aumentaron considerablemente durante la sequía como resultado del detrimento en la dilución y el aumento en la evaporación y los flujos subterráneos. Aunque el amonio y los nitritos presentaron altas variaciones, sus concentraciones no excedieron el límite para el normal desarrollo de la biota $(0.25 \mathrm{mg} / \mathrm{l})$. En la figura 3 se muestran las variables que debido a su alta fluctuación temporal (representada por el coeficiente de variación), fueron las responsables de la discriminación en el ordenamiento presentado en la figura 4.

Aunque los cambios temporales de las variables físicas y químicas fueron consistentes entre las dos zonas de estudio, se presentaron diferen-

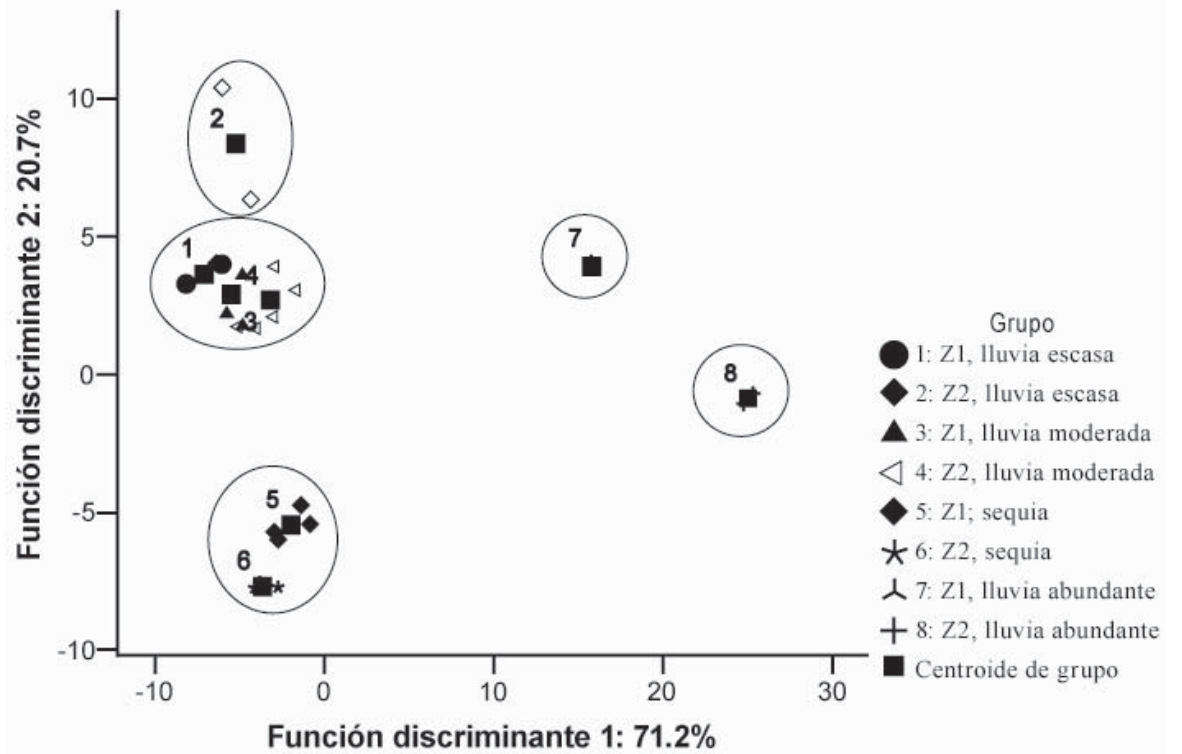

Figura 4. Funciones discriminantes canónicas entre las zonas de muestreo (Z1 y Z2) de la quebrada Potrerillos (Cauca-Colombia) y las épocas hidrológicas, con base en registros físicos y químicos hídricos. Canonical discriminant functions between sampling zones (Z1 y Z2) of the Potrerillos stream (Cauca-Colombia) and the hydrological periods based on physical and chemical water records. 
Tabla 2. Sinopsis taxonómica y abundancia relativa de los macroinvertebrados colectados en las zonas 1 y 2 de la quebrada Potrerillos (Cauca-Colombia). Taxonomic synopsis and relative abundance of macroinvertebrates collected in zones 1 and 2 of the Potrerillos stream (Cauca-Colombia).

\begin{tabular}{|c|c|c|c|c|c|c|c|c|c|}
\hline \multirow{2}{*}{ Orden/Familia } & \multirow{2}{*}{ Género o morfotipo } & \multicolumn{4}{|c|}{ Zona 1} & \multicolumn{4}{|c|}{ Zona 2} \\
\hline & & 1 & 2 & 3 & 4 & 1 & 2 & 3 & 4 \\
\hline \multicolumn{10}{|l|}{ Coleoptera } \\
\hline \multirow[t]{3}{*}{ Elmidae } & Cylloepus & 3.52 & 3.23 & 0.93 & 2.84 & 4.52 & 3.65 & 0 & 2.23 \\
\hline & Macrelmis & 0.46 & 0 & 0 & 0.65 & 0 & 0 & 0 & 0 \\
\hline & Phanocerus & 0.23 & 0.15 & 0 & 0 & 0 & 0 & 0 & 0 \\
\hline Dryopidae & Pelonomus & 0 & 0 & 1.28 & 0 & 0.60 & 0.75 & 0 & 0 \\
\hline Limnichidae & Eulimnichus & 0 & 0 & 0.21 & 0 & 0 & 1.49 & 0 & 0 \\
\hline Chrysomelidae & Galerucella & 0 & 0.19 & 0 & 0 & 0 & 0 & 0 & 0 \\
\hline Psephenidae & Psephenops & 7.09 & 9.57 & 7.28 & 1.54 & 13.46 & 12.13 & 1.52 & 2.81 \\
\hline Ptilodactylidae & Anchytarsus & 2.01 & 0.35 & 0.43 & 0.64 & 0 & 1.10 & 0 & 0.51 \\
\hline \multirow[t]{3}{*}{ Hydrophilidae } & Hydrobius & 0 & 0 & 0 & 1.98 & 0 & 0 & 0.76 & 4.14 \\
\hline & Berosus & 0 & 0 & 0 & 2.83 & 0 & 0.30 & 2.17 & 1.96 \\
\hline & Tropisternus & 0 & 0.15 & 0.84 & 0.00 & 0 & 0 & 5.08 & 0 \\
\hline \multicolumn{10}{|l|}{ Diptera } \\
\hline Simuliidae & Simulium & 7.59 & 14.65 & 0 & 13.71 & 4.98 & 1.52 & 0 & 1.15 \\
\hline Stratiomyidae & Odontomyia & 0 & 0.82 & 0 & 0 & 0 & 0 & 0 & 0 \\
\hline \multirow[t]{2}{*}{ Tipulidae } & Hexatoma & 0 & 0 & 0 & 0.28 & 0 & 0 & 0 & 0 \\
\hline & Limonia & 0 & 0 & 0 & 0 & 0 & 0 & 0 & 0 \\
\hline \multirow[t]{3}{*}{ Chironomidae } & Pentaneurini & 5.21 & 9.42 & 4.23 & 4.67 & 5.30 & 6.19 & 0.54 & 25.64 \\
\hline & Tanipodinae Mf 1 & 0 & 0.74 & 0 & 1.81 & 0 & 4.06 & 5.30 & 9.24 \\
\hline & Tanipodinae Mf 2 & 1.95 & 0.19 & 0 & 0 & 3.03 & 0 & 0 & 0 \\
\hline Chironomidae & Chironomus & 0.69 & 1.17 & 1.12 & 0 & 11.04 & 4.35 & 0.54 & 1.01 \\
\hline Dixidae & Dixella & 0 & 0 & 0.56 & 0.33 & 0 & 0.25 & 0 & 0 \\
\hline Ceratopogonidae & Alluaudomyia & 0 & 0 & 0.21 & 0.56 & 0 & 0 & 0 & 0 \\
\hline Culicidae & Culex & 0 & 0 & 0 & 0 & 0 & 0 & 4.46 & 0 \\
\hline \multicolumn{10}{|l|}{ Ephemeroptera } \\
\hline Oligoneuriidae & Lachlania & 0.23 & 0.53 & 0 & 0 & 0 & 0 & 0 & 0 \\
\hline \multirow[t]{2}{*}{ Leptophlebiidae } & Thraulodes & 16.59 & 10.93 & 0 & 2.91 & 8.52 & 12.87 & 0 & 8.51 \\
\hline & Terpides & 1.44 & 0 & 0 & 0.00 & 2.08 & 0 & 0 & 0 \\
\hline \multirow[t]{2}{*}{ Leptohyphidae } & Leptohyphes & 11.37 & 10.52 & 2.90 & 16.96 & 18.52 & 10.68 & 2.27 & 18.28 \\
\hline & Tricorythodes & 2.03 & 6.30 & 0 & 0 & 0 & 0 & 0 & 0 \\
\hline \multicolumn{10}{|l|}{ Lepidoptera } \\
\hline Pyralidae & Parargyractis? & 0.22 & 0.49 & 0 & 0 & 1.29 & 0.77 & 0 & 0.57 \\
\hline \multicolumn{10}{|l|}{ Trichoptera } \\
\hline Helicopsychidae & Smicridea & 0.00 & 0.50 & 0.71 & 0.56 & 0 & 0 & 0.97 & 0 \\
\hline \multirow[t]{2}{*}{ Hydropsychidae } & Leptonema & 8.67 & 8.83 & 0 & 14.98 & 10.85 & 17.68 & 0 & 7.71 \\
\hline & Helicopsyche & 0.72 & 1.52 & 0 & 0 & 3.47 & 5.96 & 0 & 0 \\
\hline \multirow[t]{2}{*}{ Philopotamidae } & Chimarra & 1.07 & 1.16 & 0 & 0 & 0 & 0 & 0 & 0 \\
\hline & Oecetis & 0.25 & 0.46 & 0 & 0.28 & 0 & 0 & 0 & 0 \\
\hline \multicolumn{10}{|l|}{ Megaloptera } \\
\hline Corydalidae & Corydalus & 1.87 & 2.87 & 0 & 0 & 1.19 & 5.12 & 0 & 2.43 \\
\hline \multicolumn{10}{|l|}{ Odonata } \\
\hline Libellulidae & Mf 1 & 2.45 & 0.84 & 0.56 & 4.98 & 0 & 0.78 & 0.43 & 4.32 \\
\hline & Macrothemis & 1.67 & 0 & 4.45 & 2.61 & 0 & 0 & 0 & 0 \\
\hline & Dythemis & 2.87 & 1.02 & 0 & 4.03 & 0 & 0 & 0 & 2.47 \\
\hline & Tramea & 0 & 0 & 0 & 0 & 0 & 0 & 0 & 0 \\
\hline Coenagrionidae & Telebasis & 0.65 & 0.56 & 0 & 0 & 0 & 0 & 0 & 0 \\
\hline & Argia & 2.87 & 1.91 & 1.48 & 4.84 & 2.12 & 2.41 & 3.79 & 4.87 \\
\hline Calopterygidae & Hetaerina & 7.01 & 6.48 & 0 & 1.96 & 0.60 & 4.31 & 0 & 0 \\
\hline Gomphidae & Aphylla & 0 & 0 & 0 & 0 & 0 & 0 & 0 & $\begin{array}{c}0 \\
\text { (cont.) }\end{array}$ \\
\hline
\end{tabular}


Tabla 2. (cont.)

\begin{tabular}{|c|c|c|c|c|c|c|c|c|c|}
\hline \multirow{2}{*}{ Orden/Familia } & \multirow{2}{*}{ Género o morfotipo } & \multicolumn{4}{|c|}{ Zona 1} & \multicolumn{4}{|c|}{ Zona 2} \\
\hline & & 1 & 2 & 3 & 4 & 1 & 2 & 3 & 4 \\
\hline \multicolumn{10}{|l|}{ Hemiptera } \\
\hline \multirow[t]{2}{*}{ Naucoridae } & Pelocoris & 3.70 & 0.88 & 0.14 & 0 & 3.85 & 0.51 & 0 & 0 \\
\hline & Limnocoris & 1.62 & 0.70 & 0 & 1.18 & 4.57 & 3.13 & 0 & 0 \\
\hline \multirow[t]{2}{*}{ Veliidae } & Microvelia & 0 & 0 & 0 & 0 & 0 & 0 & 0.32 & 0 \\
\hline & Rhagovelia & 0 & 0 & 6.36 & 0 & 0 & 0 & 2.27 & 0 \\
\hline Hebridae & Hebrus & 0 & 0.35 & 0 & 0 & 0 & 0 & 0 & 0 \\
\hline Belostomatidae & Lethocerus & 0 & 0 & 0 & 0 & 0 & 0 & 0 & 0 \\
\hline \multirow[t]{2}{*}{ Gerridae } & Trepobates & 0 & 0 & 0 & 0 & 0 & 0 & 0.11 & 0 \\
\hline & Limnogonus & 0 & 0 & 0 & 0 & 0 & 0 & 4.66 & 0 \\
\hline Glossiphoniiformes & Mf 1 & 0 & 0 & 0 & 6.50 & 0 & 0 & 0 & 0 \\
\hline \multicolumn{10}{|l|}{ Basommatophora } \\
\hline Physidae & Physa cubensis & 1.55 & 0.55 & 4.57 & 0.41 & 0 & 0 & 11.14 & 1.55 \\
\hline Lymnaeidae & Lymnaea columella & 0.43 & 0.86 & 0.92 & 0 & 0 & 0 & 0.11 & 0 \\
\hline Planorbidae & Biomphalaria & 0.68 & 0 & 25.98 & 2.84 & 0 & 0 & 0.11 & 0.62 \\
\hline \multicolumn{10}{|l|}{ Neogastropoda } \\
\hline Thiaridae & Melanoides tuberculata & 1.28 & 1.14 & 35.79 & 3.12 & 0 & 0 & 53.44 & 0 \\
\hline
\end{tabular}

cias significativas en las concentraciones de las variables químicas y en las mediciones de caudal entre dichas zonas. De esta manera, en el análisis discriminante se encontró que la primera función explica el $71.2 \%$ de la varianza de los grupos 1 , $3,4,7$ y 8 , y la segunda función el $20.7 \%$ de la varianza para los grupos 2,5 y 6 . La discriminación de la función uno respondió sobre todo a la variación del caudal en las dos zonas durante las épocas de lluvia escasa y moderada, en las cuales hubo alta similitud en la composición y abundancia de los macroinvertebrados. La ordenación de la función dos se relacionó con el incremento de los nitratos durante las épocas de lluvia escasa (Z2) y sequía (Z1 y Z2). Además también influyeron las variaciones en las concentraciones de calcio y dureza total (Figs. 3 y 4). Sin embargo, considerando el bajo porcentaje de casos correctamente clasificados mediante validación cruzada (25\%) no se puede asegurar la capacidad predictiva del análisis.

\section{Composición y estructura de la comunidad de macroinvertebrados}

Fueron colectados en total 3417 individuos en la quebrada Potrerillos, distribuidos en 3 phyla, 3 clases, 11 órdenes, 37 familias y 55 géneros. El orden de insectos más rico fue Coleoptera $(11$ y
8 géneros en las zonas 1 y 2 respectivamente), seguido por Diptera (10 y 8 morfotipos y géneros), Odonata (6 y 7 géneros) y Ephemeroptera (5 y 4 géneros) (Tabla 2).

De las 30 familias registradas en la Z1 las más abundantes fueron: Leptohyphidae (12.75\%), Hydropsychidae (9.82 \%), Leptophlebiidae (8.18\%), Chironomidae subfamilia Tanypodinae $(8.0 \%)$, Thiaridae $(7.89 \%)$, Simuliidae $(7.66 \%)$ y Psephenidae $(7.12 \%)$. En la Z2, de un total de 27 familias, la más abundante fue Chironomidae (18.54\%), seguida de Leptohyphidae (15.06\%), Hydropsychidae (12.78\%), Thiaridae $(8.73 \%)$ y Psephenidae $(6.80 \%)$ (Tabla 2).

En términos de composición faunística, las dos zonas de muestreo fueron $80.4 \%$ similares. Del total de 55 géneros y/o morfotipos registrados entre ambas zonas, 37 fueron comunes, 12 se registraron únicamente en la Z1 y 6 sólo en la Z2.

\section{Análisis espaciotemporal de la comunidad de macroinvertebrados}

Los muestreos tuvieron una eficiencia promedio de $73 \%$ (rango $62-82 \%$ ). Aunque no se alcanzó el promedio óptimo de taxa esperados (85\%) la curva de acumulación fue asintótica, por lo que se considera que el muestreo realizado fue representativo. 
La Z1 presentó los mayores valores de riqueza (47 géneros) y abundancia absoluta total (1902 ind.), en la $\mathrm{Z} 2$ dichos valores fueron 37 y 1515 , respectivamente (Tabla 2). Estos registros se relacionan con la disponibilidad de sustratos y la mayor heterogeneidad de hábitats de la Z1 como consecuencia del alto aporte de hojarasca, lo que posibilita mayor probabilidad de encontrar refugio ya sea durante los eventos de inundación del lecho fluvial o durante la sequía.

En relación con el régimen pluviométrico se presentaron importantes variaciones. En el primer semestre del año, correspondiente a las épocas de lluvia escasa y moderada, y aún en el inicio de la sequía (julio y agosto), la Z1 presentó la mayor diversidad del año $\left(H^{\prime}\right)$, la cual osciló entre 2.1 y 2.4 nats/ind; igualmente sucedió en la Z2, donde los registros estuvieron entre 1.7 y 2.1 nats/ind. En la sequía, principalmente en septiembre e inicios de octubre, la Z2 fue menos diversa (entre 0.4 y 1.1) con respecto a la Z1 (1.0 y 1.3). En ambas zonas, en el periodo de lluvias abundantes, se incrementó nuevamente la diversidad (Z1: entre 1.8 y 1.9 ; Z2: entre 0.8 y 0.9 ). Este mismo patrón de variación se observó para la riqueza y la equidad (Fig. 5). Por ello, las pruebas de Kruskal y Wallis -efectuadas para la riqueza, la diversidad y la dominancia- mostraron diferencias significativas entre sitios y épocas hidrológicas $(\alpha \leq 0.01)$. La equidad en ambas zonas fue constante de enero a agosto registrando valores promedio de 0.9 , y aunque en septiembre con la aparición de los pozos esta variable dismi-

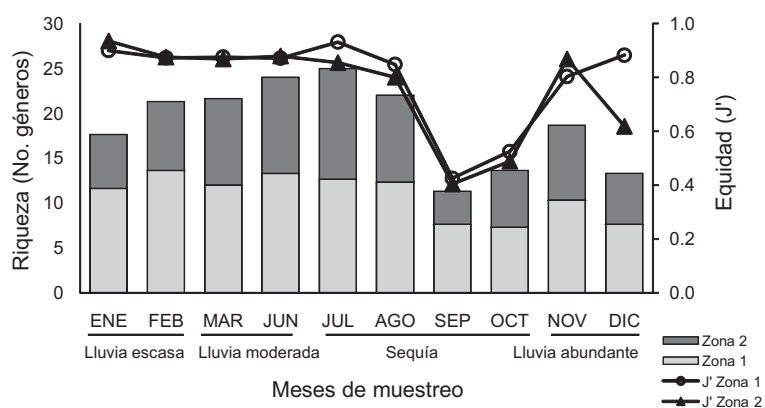

Figura 5. Variación espacial y temporal de la riqueza y la equidad $\left(J^{\prime}\right)$ de la comunidad de macroinvertebrados en la quebrada Potrerillos (Cauca-Colombia). Spatial and temporal variation of richness and evenness $\left(\mathrm{J}^{\prime}\right)$ of the macroinvertebrates community in the Potrerillos stream (Cauca-Colombia).
Tabla 3. Significancia de las pruebas de Kruskal-Wallis para los estimadores estadísticos de la comunidad de macroinvertebrados acuáticos entre los grupos (8 en total) que representan épocas hidrológicas y sitios de muestreo en la quebrada Potrerillos (Cauca-Colombia). Significance of Kruskal-Wallis tests for the statistical estimators of aquatic macroinvertebrates community between groups ( 8 in total) representing hydrological seasons and sampling sites in Potrerillos stream (CaucaColombia).

\begin{tabular}{lcl}
\hline $\begin{array}{l}\text { Estimativos } \\
\text { comunitarios }\end{array}$ & H $(\boldsymbol{n}=\mathbf{5 7})$ & $\boldsymbol{\alpha}$ \\
\hline Riqueza & 21.55 & $0.0030^{* * *}$ \\
Diversidad $\left(H^{\prime}\right)$ & 21.61 & $0.0030 * *$ \\
Equidad $(J)$ & 8.12 & 0.32 \\
Dominancia $(D)$ & 19.84 & $0.0059 * *$ \\
\hline
\end{tabular}

$\alpha<0.05^{* *}, \quad \alpha<0.01 * * *, \alpha<0.001$

nuyó hasta 0.4 (Fig. 5) -dada la desaparición de algunos taxa, la disminución de la abundancia en otros y la dominancia de Melanoides tuberculata [Thiaridae (Müller, 1774)]- no se encontraron diferencias significativas de equidad entre sitios ni entre épocas hidrológicas (Tabla 3).

Los resultados encontrados con el índice de Estabilidad de Dubois muestran que tanto la abundancia como las variables físicas y químicas fueron relativamente estables entre enero y junio e inestables entre agosto y diciembre (Fig. 6). En

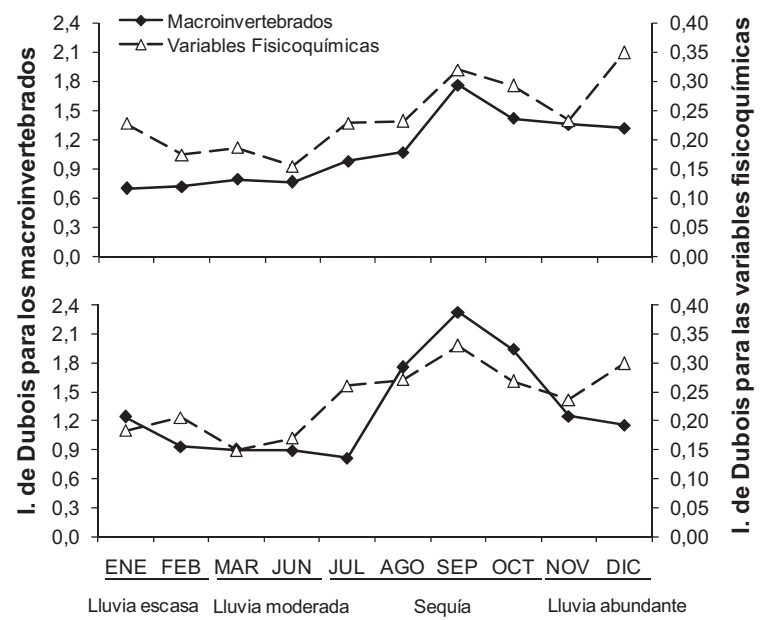

Figura 6. Variación mensual del Índice de Estabilidad de Dubois para las zonas 1 y 2 de la quebrada Potrerillos (CaucaColombia), basado en la abundancia de macroinvertebrados acuáticos y las variables físicas y químicas hídricas. Monthly variation of Dubois' Stability Index for zones 1 and 2 of the Potrerillos stream (Cauca-Colombia), based on the abundance of aquatic macroinvertebrates and the physical and chemical water variables. 
la figura 6 se observa además una relación directa entre los períodos de estabilidad y fluctuación de las variables consideradas.

Las variables abióticas que influyeron significativamente sobre la abundancia de los macroinvertebrados en ambas zonas de muestreo, se presentan en la siguiente ecuación $\left(r^{2}=0.92\right.$, $F_{6,13}=23.86, \alpha<0.001, n=20$ ).

Ln $($ I. Dubois de MA) $=2.757+0.129 * \ln$ (dureza total) $+0.189 * \ln$ (temperatura del agua) $+0.107 * \ln$ (dureza carbonácea) $+0.143 *$ $* \ln$ (caudal) $+0.075 * \ln$ (gas carbónico) $-0.057 * \ln$ (nitratos).

La significancia fue de 0.009 para la dureza total y el $\mathrm{CO}_{2} ;<0.001$ para la temperatura del agua, la dureza carbonácea y el caudal; y de 0.034 para los nitritos.

\section{DISCUSIÓN}

\section{Efecto de los cambios hidrológicos}

Si bien se encontró que aunque la sequía con la consecuente disminución y cese del flujo hídrico fue el factor influyente en los cambios anuales de las variables físicas y químicas y en la variación en la composición y estructura de los macroinvertebrados, no se puede considerar que este período hidrológico sea un evento catastrófico para la comunidad en general, ya que aunque se obtuvieron cambios significativos en su estabilidad (Fig. 6), la comunidad recuperó su estructura a medida que transcurrieron las lluvias y se inundó nuevamente el canal. Este comportamiento es típico de ecosistemas estacionales como el estudiado (Boulton, 2003; Brock et al., 2003).

En este estudio las variaciones de abundancia y riqueza de los macroinvertebrados estuvieron relacionadas con los cambios de caudal, los cuales ligados a las variaciones de los factores climáticos (e.g. temperatura y precipitación) afectaron variables físicas (e.g. temperatura hídrica y conductividad) y químicas (e.g. dureza total y carbonácea, calcio y nitratos) de los hábitats, que a su vez también influyeron en la composición y estructura de la comunidad (Fig. 4). En relación con la organización jerárquica del paisaje, el caudal opera al nivel de la cuenca e influye en variables del hábitat, tales como el establecimiento de rápidos y pozos; y del microhábitat, como el coeficiente de rugosidad y el número de Froud (Statzner \& Higler, 1985; 1986). Aunque estas últimas no fueron estimadas en este estudio, han sido consideradas de importancia capital tanto en ambientes perennes como en intermitentes (Lamouroux et al., 2004).

\section{Respuesta de la comunidad}

En relación con ecosistemas intermitentes prístinos de Australia, donde se reportan 88 especies (Robson et al., 2005) y con ecosistemas africanos, en los que se registran 175 especies (Arab et al., 2004), en este trabajo se encontraron 55 géneros, desconociéndose la riqueza de especies. A pesar de las discrepancias en la riqueza total de estos lugares y considerando las diferencias inherentes a cada uno de ellos, dichos estudios coinciden en que entre los órdenes con mayor riqueza de especies figuran Coleoptera y Diptera en Australia, África y Colombia. Además, fueron codominantes, Trichoptera en Australia, Ephemeroptera y Hemiptera en África, y Odonata en Colombia. Los autores identificaron como los grupos menos diversos a Ephemeroptera y Plecoptera en Australia, a Odonata, Trichoptera y Plecoptera en África, y en Colombia a Lepidoptera, Megaloptera y Glossiphoniforme (taxa no reportados para otras regiones).

La riqueza sobresaliente de Coleoptera y Diptera durante todos los períodos hidrológicos; y de Odonata, Trichoptera, Ephemeroptera y Hemiptera en segundo plano (Fig. 7), respalda los postulados sobre la resistencia de ciertas especies de estos grupos ante las alteraciones del flujo hídrico y los hábitats, y su alto potencial como colonizadores (Arab et al., 2004, Robson et al., 2005). No obstante, Schneider \& Frost (1996) plantean que la persistencia de muchas especies en los sistemas temporales se debe más a las adaptaciones de sus historias de vida y a las interacciones con otras especies que a procesos aleatorios tales como colonización o extinción. En este sentido se resalta que aunque la mayoría de los organismos permanentes en la quebrada tienen 


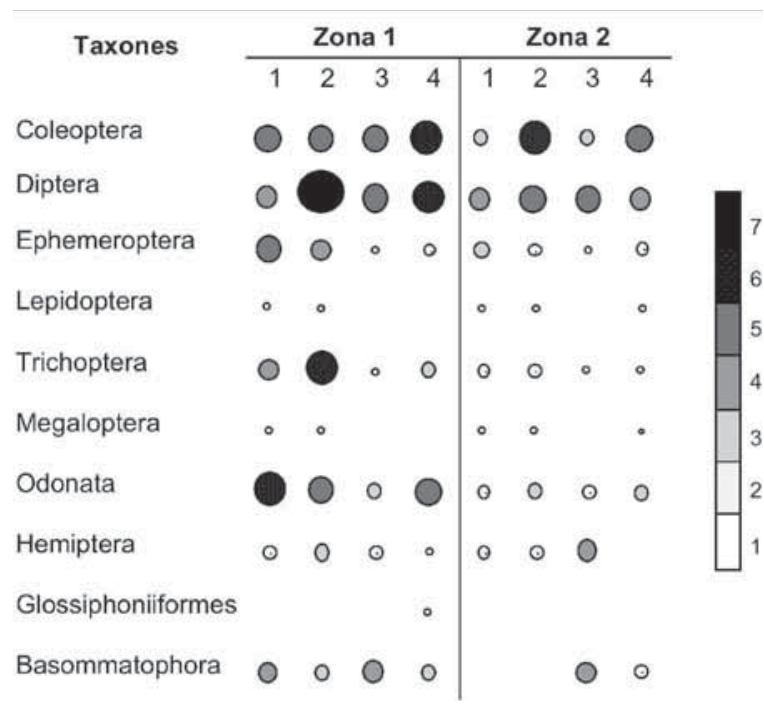

Figura 7. Cambios en la riqueza de órdenes en dos zonas de muestreo en la quebrada Potrerillos (Cauca-Colombia), en relación con cambios hidrológicos. Los círculos grandes y oscuros representan alta riqueza, mientras que los pequeños y más claros indican valores bajos, en un rango de 0-7 (1. Lluvia escasa; 2. Lluvia moderada; 3. Sequía; 4. Lluvia abundante). Changes in orders richness in two sampling zones in the Potrerillos stream (Cauca-Colombia) in relation to hydrological changes. The big dark circles represent high richness, while the small and lighter ones indicate low values, in a 0-7 range (1. Scant rain; 2. Moderate rain; 3. Drought; 4. Abundant rain).

estadios alados y acuáticos, los gasterópodos que contribuyeron significativamente en abundancia (Tabla 2) y riqueza (Fig. 7), son organismos netamente acuáticos. Tal es el caso de $M$. tuberculata cuya reproducción ocurrió durante la época de lluvias moderadas y la eclosión de los huevos durante la sequía, aumentando así exponencialmente la población. Además, el incremento del caudal ocasionó una redistribución de la población quebrada abajo y la aparición de nuevas colonias, estrategia consistente con lo reportado para la misma especie en ecosistemas templados por Pointer et al. (1993). El incremento mencionado generó durante la sequía una inestabilidad en la comunidad manifestada como un pico correspondiente a un periodo de fluctuación en el índice de Dubois (Fig. 6).

Por otro lado, el hallazgo durante la sequía y en ambas zonas de muestreo de individuos de M. tuberculata y Anchytarsus vivos, enterrados a una profundidad aproximada de $30 \mathrm{~cm}$, res- palda el planteamiento de Boulton (2003) acerca de la utilización de la zona hiporreica como refugio durante dicha época. No obstante, es importante resaltar que no se encontraron Anchytarsus en el agua de los pozos como sí ocurrió con M. tuberculata. Este comportamiento no ha sido reportado en otras zonas tropicales ni templadas, lo cual amplía el conocimiento de las posibles estrategias de supervivencia de insectos y caracoles en estos ecosistemas. Sin embargo, comúnmente se ha observado que invertebrados grandes con cuerpos rígidos que escavan o se alimentan en zonas de depósito o en sedimentos finos, son más comunes en sustratos suaves con baja rugosidad. Estos organismos proveen además cuidado parental a los juveniles y pueden entrar en diapausa o dormancia para resistir los disturbios (Lamouroux et al., 2004).

Con el inicio de las lluvias abundantes y la inundación del canal, la riqueza taxonómica nuevamente se incrementa, en este caso debido a la colonización de Leptonema, Simulium, Corydalus, Dythemis y Thraulodes. Los mecanismos de colonización de estos taxa no están completamente esclarecidos. Gasith \& Resh (1999) los atribuyen a sus estadios alados, pero Williams \& Smith (1996), luego de trabajos experimentales de recolonización a nivel de microhábitats con diferentes combinaciones de sustratos, caudal, alimento y luz, concluyeron que los controles de la colonización serían múltiples factores abióticos, bióticos, autóctonos y alóctonos. En este estudio, además de los estadios alados, la distribución de los taxa por tramo y la oferta trófica, relacionada con las actividades antrópicas propias de cada zona y la cobertura vegetal presente, estarían entre las principales características que explicarían, no sólo la rápida colonización después de un disturbio sino también la estabilidad de la comunidad. Adicionalmente, se ha reportado en zonas templadas que Leptonema y Simulium pueden sobrevivir ante eventos climáticos extremos, como la sequía y el congelamiento de los ríos, entrando en criptobiosis, específicamente en anhidrobiosis (McCafferty \& Provoncha, 1978).

Además, entre los taxa colectados en las épocas de lluvia pero ausentes durante la sequía, sobresalieron por su abundancia Cylloepus, $M a$ - 
crelmis, Phanocerus, Simulium, Tanypodinae, Thraulodes, Leptonema, Oecetis, Dythemis, Hetaerina y Limnocoris (Tabla 2). Estos son organismos asociados con altos requerimientos de oxígeno disuelto y poca concentración de materia orgánica en descomposición (Roldán \& Ramírez, 2008), características que cambian al desaparecer el flujo de agua constante.

A pesar que en este estudio no se establecieron ni los hábitos tróficos, ni los hábitos de vida, ni las relaciones intra e inter-específicas, se ha planteado que la estabilidad no es un efecto puro de las especies (i.g. efecto de la diversidad), sino que depende de las respuestas bióticas específicas de los organismos en relación con los diversos factores abióticos que afectan sus hábitats (Badii \& Ruvalcaba, 2007), lo cual es más acertado para establecer dicha estabilidad y su fluctuación. Sin embargo, ha de considerarse que esta investigación es una aproximación al igual que un referente respecto a lo que podría suceder con la diversidad de una comunidad desde el punto de vista de uno de los factores que afectan su estabilidad: la estacionalidad de las lluvias. Además, en este trabajo se refleja la importancia en la conservación de las zonas ribereñas para el establecimiento y la estructuración de las comunidades de macroinvertebrados. Dichas zonas son áreas terrestres transicionales que se extienden desde las orillas de los cuerpos de agua a las orillas de las comunidades terrestres y que por su posición, integran las interacciones del sistema acuático y el componente terrestre del paisaje. Son además ambientes dinámicos caracterizados por poseer regímenes energéticos fuertes, heterogeneidad sustancial de hábitats, diversidad de procesos ecológicos y gradientes multidimensionales. Por este dinamismo y su acción de tipo buffer, pueden reducir los flujos de sedimentos, nutrientes ( $\mathrm{N}$ y $\mathrm{P}$ especialmente) y agroquímicos provenientes de fuentes difusas, tierras de cultivo y asentamientos urbanos. Igualmente pueden regular la intensidad de los flujos en concordancia con la composición y los hábitos estructurales de la vegetación asociada a la misma (Naiman et al., 2005).

En Potrerillos, y debido a que la Z1 presenta mayor cantidad y diversidad en la cobertura vegetal ribereña, cabe pensar que existe a su vez un mayor aporte de hojarasca al lecho que favorece el incremento de la riqueza y la abundancia de los organismos al aumentar la oferta trófica y la disponibilidad y heterogeneidad de hábitats y sustratos. Por ello, la hojarasca ha sido identificada por Richardson (1992) como un hábitat potencial, que no sólo aporta alimento para unas cuantas especies, sino también, refugio contra predadores y contra corrientes directas para otras, bien sea durante los eventos de inundación del lecho fluvial o durante los eventos de sequía, convirtiéndose así en microhábitats propicios para el desarrollo de redes tróficas. Una prueba más de lo anterior la constituye el que en la zona 1 se registraron los mayores valores medios para la riqueza y la densidad (10.9 taxa y 63.4 individuos, respectivamente), en relación con la zona 2 , en la que el valor medio de riqueza fue de 8.0 taxa y el de densidad de 50.5 .

\section{CONCLUSIONES}

Las hipótesis fueron corroboradas ya que efectivamente se presentaron cambios en la estructura y composición de la comunidad tanto en el espacio como en el tiempo. Sin embargo, aunque hubo diferencias en estos atributos, en el eje longitudinal la dinámica de la comunidad en función de los factores de estrés considerados fue similar entre zonas. Así, desde el punto de vista de la estabilidad, la comunidad fue resistente y resiliente. Resiliente porque cambió estructural y composicionalmente entre períodos; y resistente porque a pesar de la influencia del disturbio, especialmente durante la sequía, la comunidad se recuperó medianamente en composición y altamente en su diversidad. No obstante, este estudio es insuficiente para establecer la dinámica y la estabilidad reales de la comunidad ya que se realizó bajo la influencia del ciclo de El Niño y no se cuenta con registros en períodos en que dicha influencia no está presente.

En consecuencia, es importante realizar estudios a largo plazo en los ecosistemas secos tropicales (terrestres y acuáticos), tanto los que están fuera del proceso de desertificación, como los que se encuentran en camino y dentro de ella. 
El acelerado cambio y la pérdida constante de ecosistemas y hábitats a causa de la industrialización, la globalización y los fenómenos "naturales" (producto de actividades antropogénicas) requieren mayores y mejores investigaciones e intervenciones sobre los recursos naturales. Conocer las historias de vida de los organismos y su relación con los cambios naturales de sus ecosistemas es una de las principales herramientas para formular y desarrollar los planes de conservación, preservación y utilización razonable de los bienes y servicios que los ecosistemas brindan.

\section{AGRADECIMIENTOS}

Agradecemos en la Universidad del Cauca a los estudiantes del énfasis en Recursos Hidrobiológicos Continentales del programa de Biología año 2006 por su colaboración en campo, en especial a Nicolás; gracias a Fernando A. Muñoz por la elaboración del mapa; y a la Vicerrectoría de Investigaciones por el soporte logístico. Agradecemos igualmente, a dos revisores anónimos por sus comentarios y sugerencias, los cuales contribuyeron a mejorar la versión final del manuscrito.

\section{BIBLIOGRAFÍA}

ARAB, A., S. LEK, A. LOUNACI \& S. PARK. 2004. Spatial and temporal patterns of benthic invertebrate communities in an intermittent river (North Africa). Ann. Limnol.-Int. J. Lim., 40(4): 317-32.

BADII, M. H. \& I. RUVALCABA. 2007. Sustentabilidad en función de estabilidad y complejidad. Daena: International Journal of Good Conscience, 2(1): 71-88.

BONADA, N. 2003. Ecology of the macroinvertebrate communities in mediterranean rivers at different scales and organization levels. PhD Dissertation, University of Barcelona, Barcelona, Spain. 355 pp.

BOULTON, A. J. 2003. Parallels and contrasts in the effects of drought on stream macroinvertebrate assemblages. Freshwater Biology, 48: 1173-1185.

BROCK, M. A., D. L. NIELSEN, R. J. SHIEL, J .D. GREEN \& J. D. LANGLEY. 2003. Drought and aquatic community resilience: the role of eggs and seeds in sediments of temporary wetlands. Freshwater Biology, 48: 1207-1218.

COLWELL, R. K. 1997. EstimateS: Statistical estimation of species richness and shared species from samples. Version 6. User's Guide and application published at: http://viceroy.eeb.uconn.edu/estimates.

COVICH, A. P., T. A. CROWL \& F. N. SCATENA. 2003. Effects of extreme low flows on freshwater shrimps in a perennial tropical stream. Freshwater Biology, 48(7): 1199-1206.

DÍAZ, J. P., G. F. ORTIZ \& G. I. NAUNDORF. 2008. Caracterización de la comunidad de algas perifíticas en la quebrada "Potrerillos", río intermitente en el municipio del Patía, Departamento del Cauca. Memorias VIII Seminario Colombiano de Limnología y I Reunión Internacional de Cuencas Bajas y Zonas Estuarinas. Santiago de Cali, Colombia: 16.

FERNÁNDEZ, H. R. \& E. DOMÍNGUEZ. 2001. Macroinvertebrados Bentónicos Sudamericanos: una guía práctica. Tucumán, Argentina: Serie Investigaciones de la Universidad Nacional de Tucumán; Subserie: Ciencias Exactas y Naturales. 282 pp.

GASITH, A. \& V. H. RESH. 1999. Streams in Mediterranean climate regions: Abiotic influences and biotic responses to predictable seasonal events. Annual Review of Ecology and Systematics, 30: 51-82.

GUISANDE, G. C., B. A. FELPETO, E.I MANEIRO, R. I. ALARCÓN, C. A. R. VERGARA \& L. A. VAAMONDE. 2006. Tratamiento de datos. España: Díaz de Santos. 356 pp.

HALWAS, K. L., M. CHURCH \& J. S. RICHARDSON. 2005. Benthic assemblage variation among channel units in high-gradient streams on Vancouver Island, British Columbia. J. N. Am. Benthol. Soc., 24(3): 478-494.

HOLDRIDGE, L. 1978. Ecología basada en las zonas de vida. San José, Costa Rica: Instituto Interamericano de Ciencias Agrícolas. 286 pp.

HUMPHRIES P. \& D. S. BALDWIN. 2003. Drought and aquatic ecosystems: an introduction. Freshwater Biology, 48: 1141-1146.

INSTITUTO E HIDROLOGÍA, METEOROLOGÍA Y ESTUDIOS AMBIENTALES (IDEAM). 1999. Caracterización climática del Macizo Colombiano. En: El Macizo Colombiano y su área de influencia. Ministerio del Medio Ambiente (ed.): 20-21. Ministerio del Medio Ambiente, Santa Fé de Bogotá, Colombia. Disponible en: http://www.ideam.gov. co/publica/Macizo/introducci \%C3\%B3n.pdf 
INSTITUTO E HIDROLOGÍA, METEOROLOGÍA Y ESTUDIOS AMBIENTALES (IDEAM). 2007. Seguimiento de El Niño en Colombia. Informe No 3.7 pp. Disponible en: http://www.fenalce.org/ archivos/seguimiento3.pdf

JUNK, W. J. \& K. M. WANTZEN. 2004. The Flood Pulse Concept: New Aspects Approaches and Applications-an Update. Proceedings of the Second International Symposium on the Management of Large Rivers for Fisheries. R. L. Welcomme \& T. Petr (eds.). 2:117-140. Food and Agriculture Organization \& Mekong River Commission. FAO Regional Office for Asia and the Pacific, Bangkok.

LAKE, P. S. 2003. Ecological effects of perturbation by drought in flowing waters. Freshwater Biology, 48: 1161-1172.

LAMOUROUX, N., S. DOLÉDEC \& S. GAYRAUD. 2004. Biological traits of stream macroinvertebrate communities: effects of microhabitat, reach, and basin filters. J. N. Am. Benthol. Soc., 23: 449-466.

LEMUS, R., LTDA \& CORPORACIÓN REGIONAL DEL CAUCA (CRC). 1990. Estudio del potencial hidráulico de la Cuenca Hidrográfica de río Patía. Popayán, Colombia, 5-20.

McCAFFERTY, W. \& A. PROVONSHA. 1978. Aquatic Entomology. Boston, Massachusets: Science Books International. $448 \mathrm{pp}$.

MERRITT, R. \& K.W. CUMMINS. 1996. An introduction to the aquatic insects of North America. $3^{\text {th }}$ ed. Duduque: Kendal/Hunt. 441 pp.

MINISTERIO DE AMBIENTE, VIVIENDA Y DESARROLLO TERRITORIAL. 2004. Plan de acción nacional de lucha contra la desertificación y la sequía en Colombia -P.A.N-. Ministerio de Medio Ambiente, Vivienda y Desarrollo Territorial. Bogotá, D.C., Colombia. 137 pp. Disponible en: http://www.siac.gov.co/documentos/DOC_Portal/ DOC_Suelo/010905_plan_lucha_contra_deser.pdf

MOL J. H., D. RESIDA, J. S. RAMLAL \& C. R. BECKER. 2000. Effects of El Niño-related drought on freshwater and brackish-water fishes in Suriname, South America. Environmental Biology of Fishes, 59: 429-440.

NAIMAN, R. J., H. DÉCAMPS \& M. E. McLAIN. 2005. Riparia. Ecology and management of streamside communities. Amsterdam: Elsevier. $430 \mathrm{pp}$.

PENNAK, R. 1978. Fresh-Water Invertebrates of the United States. $2^{\text {nd }}$ ed. New York: J. Wiley \& Sons. 664 pp.
POINTER, J. P., A. THÉRON \& G. BOREL. 1993. Ecology of the introduced snail Melanoides tuberculata (Gastropoda: Thiaridae) in relation to Biomphalaria glabrata in the marshy forest zone of Guadeloupe, French West Indies. J. Moll. Stud., 59: 421-428.

POOLE, G. C. 2002. Fluvial landscape ecology: Addressing uniqueness within the river discontinuum. Freshwater Biology, 47: 641-660.

RAMÍREZ, A., P. PAABY, C. M. PRINGLE \& G. AGÜERO. Effect of habitat type on benthic macroinvertebrates in two lowland tropical streams, Costa Rica. Rev. Biol. Trop., 46: 201-213.

RIBEIRO O. L. \& S. V. UIEDA. 2005. Estrutura da comunidade de macroinvertebrados bentónicos de um riacho de serra em Itatinga, São Paulo, Brasil. Revista Brasileira de Zoologia, 22(3): 613-618.

RICHARDSON, J. S. 1992. Food, microhabitat, or both? Macroinvertebrate use of leaf accumulations in a montane stream. Freshwater Biology, 27: 169-176.

RINCON, J. \& C. CRESSA. 2000. Temporal variability of macroinvertebrate assemblages in a neotropical intermittent stream in northwestern Venezuela. Archiv für Hydrobiologie, 3: 421-432.

ROBSON, B. J., M. HOGAN \& T. FORRESTER. 2005. Hierarchical patterns of invertebrate assemblage structure in stony upland streams change with time and flow permanence. Freshwater Biology, 50: 944-953.

RODRÍGUEZ, E., M. D. HUBBARD \& W. L. PETERS. 1992. Clave para ninfas y adultos de las familias y géneros de Ephemeroptera (Insecta) sudamericanos. Biología Acuática, 16: 8-38.

ROLDÁN, G. 1988. Guía para el estudio de los macroinvertebrados acuáticos del Departamento de Antioquia. Bogotá: Fondo FEN-COLCIENCIAS. 217 pp.

ROLDÁN, G. \& J. J. RAMÍREZ. 2008. Fundamentos de limnología neotropical, $2^{\mathrm{a}}$ ed. Medellín: Accefyn, UCO, Universidad de Antioquia. 440 pp.

SCHNEIDER, D. W. \& T. M. FROST. 1996. Habitat duration and community structure in temporary ponds. J. N. Am. Benthol. Soc., 15: 64-86.

STATZNER, B. \& B. HIGLER. 1985. Questions and comments on the River Continuum Concept. $\mathrm{Ca}$ nadian Journal of Fisheries and Aquatic Sciences, 42: 1038-1044.

STATZNER, B. \& B. HIGLER. 1986. Stream hydraulics as a major determinant of benthic invertebrate zonation patterns. Freshwater Biology, 16: 127-139. 
TOMANOVA, S., E. GOITIA \& J. HELES. 2006. Trophic levels and functional feeding groups of macroinvertebrates in neotropical streams. Hydrobiologia, 556: 251-264.

TORRES, M. P., D. G. IBÁÑEZ \& E. J. VÁSQUEZ. 1992. Generalidades sobre la geología de norte del Valle del Patía. Novedades Colombianas, 1-26.

TRAMER, E. J. 1977. Catastrophic mortality of stream fishes trapped in shrinking pools. American Midland Naturalist, 97: 469-478.

VANNOTE, R. L., G. W. MINSHALL, K. W. CUMMINS, J. R. SEDELL \& C. E. CUSHING. 1980. The river continuum concept. Can. J. Fish Aquat. Sci., 37: 130-137.

WARD, J. V. \& J. A. STANFORD. 1983. The serial discontinuity concept of lotic ecosystems. In: $D y$ namics of Lotic Ecosystems. T. D. Fontaine \& S. M. Bartell (eds.): 29-42. Ann Arbor Science Publishing, Michigan, USA.

WIGGINS, G. B. 1973. A contribution to the biology of caddisflies (Trichoptera) in temporary pools. $\mathrm{Li}$ fe Sci. Contr. Royal OntarioMuseum, 88: 1-28.

WILLIAMS, D. D. 1996. Environmental constraints in temporary fresh waters and their consequences for the insect fauna. J. N. Am. Benthol. Soc., 15: 634-650.

WILLIAMS, D. D. \& M. SMITH. 1996. Colonization dynamics of river benthos in response to local changes in bed characteristics. Freshwater Biology, 36(2): 237-248. 